\title{
Bone Marrow Cells and Cardiac Repair
}

Understanding the mechanisms by which adult somatic stem cells are capable of improving cardiac function in ischemic heart disease is of fundamental biological and clinical importance ${ }^{1}$. Endothelial progenitor cells and mononuclear bone marrow cells have been administered to patients affected by acute and chronic ischemic heart failure ${ }^{2}$. These interventions have had positive outcome documenting not only the feasibility and safety of this therapeutic approach but also beneficial effects on ventricular performance. The study in the current issue of the Arquivos Brasileiros de Cardiologia unexpectedly documents that intracoronary injection of bone marrow mononuclear cells to patients suffering from Chagas cardiomyopathy is safe and may have a favorable impact on the late evolution of the cardiac disease ${ }^{3}$. The trial included 28 patients with congestive heart failure due to infection by Trypanosoma cruzi. The cell treatment resulted in improvement in ejection fraction and functional NYHA class, together with amelioration of quality of life and increased walked distance.

The pathology of Chagas cardiomyopathy differs significantly from post-infarction myopathy ${ }^{4}$; it is characterized by diffuse myocardial scarring and chronic cellular infiltrates composed of lymphocytes, plasma cells and macrophages, which indicate an ongoing active process with continuous loss of myocytes and activation of connective tissue cells and collagen deposition. These structural abnormalities of the myocardium complicate further the interpretation of the potential therapeutic efficacy of bone marrow cells (BMCs) in Chagas disease. Experimental studies in animal model of ischemic cardiomyopathy have raised several questions concerning the multiple variables involved in cardiac repair after BMC implantation ${ }^{5,6}$. And these factors apply to the management of Chagas disease as well. They include (a) the number of therapeutically effective cells which are administered, (b) the magnitude of cell death and survival of the BMCs in the hostile milieu of the damaged region of the heart, (c) the extent of BMC engraftment, and, finally, (d) the degree of growth and differentiation of the homed BMCs. An additional critical variable of BMCs is their level of plasticity, which is dictated by their ability to acquire the myocyte, and vascular smooth muscle and endothelial cell lineages ${ }^{7}$. Importantly, the injected
BMCs can contribute indirectly to cardiac regeneration by releasing a variety of peptides that exert a paracrine action on the myocardium ${ }^{8}$ and its resident cardiac progenitor cells (CPCs) ${ }^{9}$. These mechanisms are not mutually exclusive and bone marrow progenitor cells may participate directly and indirectly in the repair process. In all cases, however, progenitor cells have to engage themselves in homing into the myocardium in order to perform specific functions. These biological processes depend on a successful interaction between progenitor cell classes and tissue microenvironment ${ }^{10}$. Although the clinical trial performed by Vilas-Boas and collaborators projects an unprecedented implication of stem cell therapy for the damaged heart ${ }^{3}$, the issues raised above will have also to be addressed in Chagas disease to acquire a biological understanding of the function of BMCs in this cardiomyopathy.

The mechanisms that guide stem cell homing in the myocardium are not completely understood. Cardiac injury may be necessary for migration and long-term engraftment of stem cells in the myocardium. In the absence of tissue damage, the implanted stem cells are at growth disadvantage with respect to the endogenous stem cells ${ }^{11}$. Homing and engraftment depend on a successful interaction between progenitor cells and tissue microenvironment ${ }^{12,13}$. Distinct cell classes may be differently equipped to home to the injured heart. Unselected BMCs and CD34-enriched BMCs administered in the coronary vasculature of patients have different homing efficiency in 5-10 day-old infarcts ${ }^{14}$. Fifty to 75 minutes after delivery, only 1.3-2.6\% of unselected BMCs are found in the myocardium while $14-39 \%$ of the total radioactivity of CD34 positive BMCs is detected in the infarcted heart. Of relevance, the bio-distribution of the two progenitor cell types also differs with a preferential localization of the CD34-enriched cells in the border zone and a more broad localization of unselected BMCs in the border zone and in the infarcted region ${ }^{14}$. Thus, the utilization of a more specific BMC subset which contains the primitive cell pool could result in a more efficient engraftment and formation of cardiomyocytes and coronary vessels replacing the scarred tissue with functionally competent myocardium.

Mailing Address: Piero Anversa, M.D. • Vosburgh Pavilion, Room 302, New York Medical College, Valhalla, NY 10595 E-mail: piero_anversa@nymc.edu 
The study by Vilas-Boas and collaborators is extremely encouraging and has created the foundation for a double-blind multi-center clinical trial, which will have to be conducted in the future to prove unequivocally the therapeutic efficacy of BMCs in Chagas disease ${ }^{3}$. This is also true for acute and chronic coronary artery disease ${ }^{15}$. However, while patients are currently being enrolled in large clinical trials in Europe, the documentation of CPCs has created great expectation concerning the utilization of this new cell for the management of the human disease ${ }^{9}$. Theoretically, the most logic and potentially powerful cell to be employed for cardiac repair is the CPC. It is apparent that if the adult heart possesses a pool of primitive, undifferentiated, multipotent cells, these cells must be tested first, before more complex and unknown cells are explored. Cardiac regeneration would be accomplished by enhancing the normal turnover of myocardial cells. However, difficulties exist in the acquisition of myocardial samples in humans, and in the isolation and expansion of CPCs in quantities that can be employed therapeutically. Conversely, BMCs constitute an appealing form of cell intervention; BMCs can be easily collected from bone marrow aspirates or the peripheral blood upon their mobilization from the bone marrow with cytokines. At present, it is unknown whether CPCs and BMPCs are similarly effective in reconstituting dead myocardium or limitations exist in CPC growth and BMC transdifferentiation resulting in inadequate restoration of lost tissue. Also, BMCs may constitute a necessary initial form of intervention for the acutely failing heart whereas CPCs might be employed later during the chronic evolution of the cardiac myopathy. A particular case is Chagas disease. Uncertainty concerning the presence of Trypanosoma cruzi chronically in the failing heart raises questions on the utilization of CPCs in view of the potential localization of the parasite in this cell population. This possibility makes the study by Vilas-Boas and colleagues ${ }^{3}$ and the utilization of BMCs highly relevant for the future implementation of cell therapy in Chagas disease.

\section{REFERENCES}

1. Leri A, Kajstura J, Anversa P. Cardiac stem cells and mechanisms of myocardial regeneration. Physiol Rev. 2005;85:1373-1416.

9. Anversa P, Kajstura J, Leri A, Bolli R. Life and death of cardiac stem cells: a paradigm shift in cardiac biology. Circulation. 2006;113:1451-63.

2. Wollert KC, Drexler H. Clinical applications of stem cells for the heart. Circ Res. 2005;96:151-63.

3. Vilas-Boas F, Feitosa GS, Soares MBP, Pinho-Filho JA, Mota A, Gonçalves Almeida AJ, et al. Early results of bone marrow cell transplantation to the myocardium of patients with heart failure due to Chagas disease. Arq Bras Cardiol. 2006; 87:159-66.

4. Urbanek K, Torella D, Sheikh F, De Angelis A, Nurzynska D, Silvestri $F$, et al. Myocardial regeneration by activation of multipotent cardiac stem cells in ischemic heart failure. Proc Natl Acad Sci USA. 2005; 102:8692-7.

5. Losordo DW, DimmelerS. Therapeutic angiogenesis and vasculogenesis for ischemic disease. Part II: cell-based therapies. Circulation. 2004;109:2692-7.

6. Anversa P, Leri A, Kajstura J. Cardiac regeneration. J Am Coll Cardiol. 2006;47:1769-76.

7. Leri A, Kajstura J, Anversa P. Identity deception: not a crime for a stem cell. Physiology. 2005;20:162-8.

8. Yoon YS, Wecker A, Heyd L, Park JS, Tkebuchava T, Kusano K, et al. Clonally expanded novel multipotent stem cells from human bone marrow regenerate myocardium after myocardial infarction. J Clin Invest. 2005;115:326-38.

10. Urbanek K, Cesselli D, Rota M, Nascimbene A, De Angelis A, Hosoda $T$, et al. Stem cell niches in the adult mouse heart. Proc Natl Acad Sci USA. 2006;103:9226-31.

11. Bhattacharya D, Rossi DJ, Bryder D, Weissman IL. Purified hematopoietic stem cell engraftment of rare niches corrects severe lymphoid deficiencies without host conditioning. J Exp Med. 2006;203:73-85.

12. Peti I, Szyper-Kravitz M, Nagler A, Lahav A, Peled A, Habler L, et al. G-CSF induces stem cell mobilization by decreasing bone marrow SDF-1 and up-regulating CXCR4. Nat Immunol. 2002;3:687-93.

13. Quesenberry PJ, Colvin G, Abedi M. Perspective: fundamental and clinical concepts on stem cell homing and engraftment: a journey to niches and beyond. Exp Hematol. 2005;33:9-19.

14. Hofmann M, Wollert KC, Meyer GP, Menke A, Arseniev L, Hertenstein $B$, et al. Monitoring of bone marrow cell homing into the infarcted human myocardium. Circulation. 2005;111:2198-202.

15. Assmus B, Schachinger V, Teupe C, Britten M, Lehmann R, Dobert M, et al. Transplantation of progenitor cells and regeneration enhancement in acute myocardial infarction (TOPCARE-AMI). Circulation. 2002;106:3009-17. 